\title{
SOME UNIVERSAL SETS OF TERMS
}

BY

WALTER TAYLOR

\begin{abstract}
For every $\Pi_{2}^{1}$ class of cardinals containing 0 and 1 , there exists a finite
\end{abstract} set $T$ of terms, such that $X$ is precisely the class of cardinals in which $T$ is universal.

Around 1935, W. Sierpiński discovered [42] the remarkable fact that if $\left(f_{1}, f_{2}, \ldots\right)$ is any sequence of functions on an infinite set $E$ (i.e. $f_{i}: E \rightarrow E$ for each $i$ ), then there exist two functions $g_{1}, g_{2}: E \rightarrow E$ such that each $f_{i}$ is in the semigroup generated by $g_{1}$ and $g_{2}$ under composition of functions. In fact, according to a simplification found soon afterward by Banach [3], we may always take $g_{1}$ and $g_{2}$ so that the $f_{i}$ are given by these formulas:

$$
f_{1}(x)=g_{1} g_{2} g_{1} g_{2}(x), \quad f_{2}(x)=g_{1} g_{2}^{2} g_{1} g_{2}(x), \quad f_{3}(x)=g_{1} g_{2}^{3} g_{1} g_{2}(x), \ldots
$$

For functions of two variables, J. Loś [27] found a similar result around 1950; in fact he found an infinite sequence, of terms built from a single $g_{1}(x, y)$, which was capable of representing any sequence of operations on an infinite set. For some more historical comments, some easy proofs, and a good elementary introduction to these ideas, we refer the reader to [34].

Later Mycielski coined the term universal for (finite or infinite) sets of terms which, like the above $\left\{g_{1} g_{2}^{i} g_{1} g_{2}: i \in \omega\right\}$, can represent any sequence of functions through proper choice of the $g_{i}$ (a precise definition is in $\$ 0$ below). All known methods for constructing universal sets of terms relied on only the most basic properties of infinite sets (essentially $|E|^{2}=|E|$ ), and so all known examples were universal on all infinite sets $E$, i.e. regardless of the value of the infinite cardinality $|E|$. Mycielski suggested refining the notion to $\kappa$-universality (the above notion restricted to sets $E$ with $|E|=\kappa$ ), and then he asked whether $\kappa$-universality and $\lambda$-universality are equivalent for all infinite $\kappa$ and $\lambda$. (This question was reported in Isbell [15], and it really involved, at that time, a single term built from unary functions only-a special case of the problem which remains open today. The general form of the problem, which we are answering here, was stated some ten years later by G. McNulty on p. 205 of [31].) Here, in our main result, we answer "no" to the general question:

THEOREM. For each $\Pi_{2}^{1}$ class $X$ of cardinals with $0,1 \in X$, there exists a finite set Tof terms such that

$$
X=\{\kappa: T \text { is universal in power } \kappa\} .
$$

Received by the editors August 30, 1980.

1980 Mathematics Subject Classification. Primary 08A40, 04A05, 04A15; Secondary 18C10, 20 M20. 
Moreover, there exists a recursive function $f$ such that we may take $T=f\left(\right.$ any $\Pi_{2}^{1}$ formula defining $X$ ).

For the precise definition of " $\Pi_{2}^{1}$ class", see $\$ 0$; for this introduction it suffices to say that the class of all $\Pi_{2}^{1}$ classes is very rich and contains almost every easily conceived cardinal class, including, for instance: $\left[0, \aleph_{\alpha}\right),\{0,1\} \cup\left[\beth_{\alpha}, \infty\right),\{0,1\} \cup$ $\{\kappa: \kappa$ is inaccessible $\},\left[0,2^{\mu}\right)$, for $\alpha$ any recursive ordinal and $\mu$ the first measurable cardinal (but not $\left\{2^{\kappa_{0}}\right\}$ and not $[0, \mu)$-see [22] and [14]). For a full treatment of $\Pi_{2}^{1}$ classes see S. J. Garland [14]. It is immediate from the definitions ( $\$ 0$ ) that universality of $T$ in power $\kappa$ defines a $\Pi_{2}^{1}$ class containing 0 and 1 . Hence our answer to the Mycielski-McNulty question is "best possible" to the extent that one

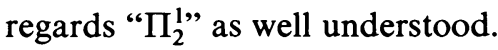

Given an object $E$ in any category with finite products, we can define the clone $C(E)$ (in Lawvere's terminology, an algebraic theory) to be the full subcategory spanned by $E$ and all its finite powers $E^{n}$. More prosaically, $C(E)$ is the family of all maps $E^{n} \rightarrow E$ (any $n$ ) together with some obvious composition operators. Thus $C(E)$ extends the endomorphism monoid $\operatorname{End}(E)$ and the automorphism group $\operatorname{Aut}(E)$. (And, like monoids and groups, the class of all clones can be described axiomatically; moreover every clone $C$ occurs, within isomorphism, in the category of all algebras with $|C|$ unary operations [37].)

One simple place to form clones is in the category of sets. Here, our result says that if $\kappa$ and $\lambda$ are cardinals which are not $\Pi_{2}^{1}$-equivalent, then $C(\kappa)$ and $C(\lambda)$ are not elementarily equivalent; in fact there is a special form of $A E$-sentence true in one but not in the other. See McKenzie [28], Shelah [38] and Pinus [36] for some results on the elementary equivalence classes of $\operatorname{End}(\kappa)$ and especially $\operatorname{Aut}(\kappa)$. In contrast to the clone situation, there do exist $\Pi_{2}^{1}$-inequivalent cardinals $\kappa$ and $\lambda$ with $\operatorname{Aut}(\kappa) \equiv \operatorname{Aut}(\lambda)$. Our results may thus be viewed as contributing to the recently developing study of the first order properties of clones, described in Baldwin and Berman [2]. (See also [53].)

The definition of universality of terms makes sense of course in any clone; one case of interest to many mathematicians has been the clone of all continuous real-valued functions of finitely many real variables, i.e. $C(R)$ in the category of continuous maps. Hilbert's thirteenth problem stimulated research which led to the discovery of various terms which are universal for this clone, including formulas to express every continuous function of three variables as a combination of continuous binary functions. For good surveys of this work of Kolmogorov, Arnol'd et al., and also the analytic clone, etc., see Lorentz [26], the introduction to Vitushkin [52], and the published solution for [34]. For clones $C(X)$ with $X$ various other topological spaces, see Taylor [49] and references given there.

$\Pi_{2}^{1}$ classes of ten arise very naturally in mathematical (particularly in algebraic) research, e.g. the class of cardinalities in which a given theory has no simple algebra, or in which it has no Jónsson algebra, etc. In $\$ 2$ we will review some of these, and in some special cases we will be able to bypass the proof of the main theorem to directly write down a set $T$ of terms which is universal in the appropriate powers. (See 2.7-2.10.) We advise the reader that this section is easier than $\S 1$, and yet these special examples really contain all the ideas of $\S 1$. 
The recursiveness assertion of the Theorem has the following Corollary, answering a question which was stated on p. 29 of [48] and really goes back to Isbell [15]. The proof is immediate from the Theorem and any of various known results about decidability, e.g. Perkins' result [35] that it is undecidable whether a finite set of identities has a model of power $\kappa_{0}$. (Also see 2.9 below.)

COROLlaRY. It is undecidable whether a finite set $T$ of terms is universal in power $\aleph_{0}$. Likewise, whether $T$ is universal in all infinite powers.

It is open whether the Theorem holds for terms built completely from unary operations, i.e. whether $\operatorname{End}(\kappa)$ carries as much information as the clone $C(\kappa)$ as far as these special $A E$-sentences are concerned. (But cf. Theorem 4 of Pinus [36] which tells us that $\operatorname{End}(\kappa) \not \operatorname{End}(\lambda)$ for $\kappa, \lambda$ second-order distinguishable cardinals.) In fact, it is even open whether a finite unary $T$ can distinguish any two infinite cardinals! The question of a unary version of the Corollary also remains open, although it has stimulated important work, and many special cases are now solved. (See the papers of Ehrenfeucht, Silberger and Valente.)

Our proofs seem to make essential use of $n$-ary operations in $T$ for arbitrarily large finite $n$. But notice that (in the infinite range) both the Theorem and its Corollary hold with $T$ restricted to terms involving a single binary operation only. We need only replace each $n$-ary operation by a suitable combination of one binary operation (in fact we may use any set of terms which is universal in all infinite powers, e.g. the above-mentioned terms of Loś). Obviously there is no such easy reduction to the unary case.

These results were announced in [50] and were supported, in part, by the N.S.F.

I thank George McNulty, Ralph McKenzie, Bill Reinhardt, Wilfrid Hodges and Gary Brenner for valuable conversations about this work, and Arthur Knoebel and Don Monk for supplying various references.

0. Definitions. Let $\varphi$ be a first order sentence with relation symbols included in $R_{1}, R_{2}, \ldots, S_{1}, S_{2}, \ldots$ and operations symbols included in $F_{1}, F_{2}, \ldots, G_{1}, G_{2}, \ldots$ Obviously the truth of the second order sentence

$$
\psi=\left(\forall R_{1} R_{2} \cdots F_{1} F_{2} \cdots\right)\left(\exists S_{1} S_{2} \cdots G_{1} G_{2} \cdots\right) \varphi
$$

in a structure $(A ; \ldots)$ depends only on the domain cardinality $\kappa=|A|$. Thus we simply say " $\kappa$ obeys $\psi$ ", in symbols $\kappa \vDash \psi$. (For this notion, the operation symbols $F_{i}, G_{i}$ are clearly dispensable, but we need them below.) Now we can state that the $\Pi_{2}^{1}$ class specified by $\psi$ is $\{\kappa: \kappa \vDash \psi\}$, and we define $X$ to be $\Pi_{2}^{1}$ iff for some $\psi X$ is the $\Pi_{2}^{1}$ class specified by $\psi$.

A set-more properly, a sequence-of terms, $T=\left(\tau_{1}, \tau_{2}, \ldots\right)$ is universal in power $\kappa$ if it can represent every sequence $\left(F_{1}, F_{2}, \ldots\right)$ of operations (of the appropriate arities) on a set of cardinality $\kappa$. More precisely, let us assume that the operation symbols appearing in $T$ are $G_{1}\left(m_{1}\right.$-ary), $G_{2}\left(m_{2}\right.$-ary $), \ldots$, and that the variables occurring in $\tau_{i}$ are $x_{1}, x_{2}, \ldots, x_{n_{i}}$. (This last assumption simplifies the definition, represents no loss of generality, and will be true of the terms constructed in the proof of Theorem.) Taking $F_{i}$ to be a new $n_{i}$-ary operation symbol for each $i$, 
we say that $T$ is universal in power $\kappa$ iff $\kappa$ obeys the sentence

$$
\left(\forall F_{1} F_{2} \cdots\right)\left(\exists G_{1} G_{2} \cdots\right) \forall x_{1} x_{2} \cdots \bigwedge_{i} \tau_{i}=F_{i}\left(x_{1}, x_{2}, \ldots\right) .
$$

(Note that this definition makes sense for infinite $T$ as well as finite.) Obviously for $\kappa>1$, any repetition in the sequence $T$ will kill universality, and rearrangements do not affect universality; thus we allow ourselves to speak of universality for $T$ a set of terms.

1. Proof of the Theorem. We are given a $\Pi_{2}^{1}$ class $X$ which is to be represented by universality of terms. Let us suppose that $X$ is defined as in $\$ 0$ by the second order sentence

$$
\psi=(\forall \cdots F \cdots R \cdots)(\exists \cdots G \cdots S \cdots) \varphi
$$

with $\varphi$ first order.

LEMMA 1. We may assume $\varphi$ is universal.

Proof. By Skolemization. Assuming $\varphi$ prenex, w.l.o.g. of the form $\forall x_{1} \exists y_{1} \forall x_{2} \exists y_{2} \ldots$, we replace all occurrences of each $y_{i}$ by a new $G_{i}\left(x_{1}, \ldots, x_{i}\right)$, and then add the $G_{i}$ 's to the list of existentially quantified operation symbols. As is well known, the satisfiability of $\varphi$ is not altered by this modification.

LEMMA 2. We may assume that $\psi$ has the form

$$
(\forall \cdots F \cdot \cdots)(\exists \cdots G \cdot \cdots)\left(\varphi_{1} \rightarrow \varphi_{2}\right)
$$

with $\varphi_{1}$ and $\varphi_{2}$ both positive universal, and with no $G$ 's appearing in $\varphi_{1}$.

(N.b. We expressly mean that no relation predicates except equality appear in $\psi$.)

Proof. Corresponding to the relations $R$ and $S$ occuring in $\psi$ we will take new operations $F_{R}$ and $G_{S}$ (of the same arity), plus new nullary operations $F_{0}, F_{1}$ and one new ternary operation $G$. We take $\varphi$ universal, as given by Lemma 1 , and moreover we make sure that $\neg$ appears in $\varphi$ only in forming the negations of atomic formulas. (In particular, $\rightarrow$ and $\perp$ do not appear.) We now form $\varphi^{*}$ from $\varphi$ by making the following alterations:

$$
\begin{aligned}
R\left(\tau_{1}, \ldots, \tau_{n}\right) & \text { becomes } F_{R}\left(\tau_{1}, \ldots, \tau_{n}\right)=F_{1} ; \\
\neg R\left(\tau_{1}, \ldots, \tau_{n}\right) & \text { becomes } F_{R}\left(\tau_{1}, \ldots, \tau_{n}\right)=F_{0} ; \\
S\left(\tau_{1}, \ldots, \tau_{n}\right) & \text { becomes } G_{S}\left(\tau_{1}, \ldots, \tau_{n}\right)=F_{1} ; \\
\neg S\left(\tau_{1}, \ldots, \tau_{n}\right) & \text { becomes } G_{S}\left(\tau_{1}, \ldots, \tau_{n}\right)=F_{0} ; \\
\neg \sigma=\tau & \text { becomes } G(\sigma, \sigma, \tau)=F_{0} \wedge G(\sigma, \tau, \tau)=F_{1} .
\end{aligned}
$$

Thus $\varphi^{*}$ is positive and universal. Now we take $\varphi_{1}$ to be the universal closure of

$$
\bigwedge_{R}\left[F_{R}\left(x_{1}, x_{2}, \ldots\right)=F_{0} \vee F_{R}\left(x_{1}, x_{2}, \ldots\right)=F_{1}\right] \text {, }
$$

and we take $\varphi_{2}$ to be the universal closure of

$$
\left(F_{0}=F_{1}\right) \vee\left[\varphi^{*} \wedge \wedge_{S}\left(G_{S}\left(x_{1}, x_{2}, \ldots\right)=F_{0} \vee G_{S}\left(x_{1}, x_{2}, \ldots\right)=F_{1}\right)\right]
$$

Obviously $\varphi_{1}$ and $\varphi_{2}$ have the required syntactic features. It remains to be seen that $\kappa \vDash \psi \leftrightarrow \kappa \vDash \psi^{\prime}$ for all cardinals $\kappa$. We are given that 0 and 1 obey $\psi$, and it is 
obvious that 0 and 1 also obey $\forall F_{0} F_{1} \cdots \exists \cdots G \cdot\left(F_{0}=F_{1}\right)$, which implies $\psi^{\prime}$. Thus we may assume $\kappa>1$. First let us assume that $\kappa \vDash \psi$. Thus we are given functions $\cdots F \cdots\left(\right.$ as in $\psi$ ), $\cdots F_{R} \cdots$ (with $R$ as in $\psi$ ), $F_{0}$ and $F_{1}$. If $F_{0}=F_{1}$ or if $\varphi_{1}$ fails, we are done, since then $\psi^{\prime}$ holds regardless of our choice of $G$ 's. Thus we will assume that $F_{0} \neq F_{1}$ and that $\varphi_{1}$ holds. Now for each symbol $R$ of $\psi$, define a relation

$$
R=\left\{\vec{x}: F_{R}(\vec{x})=F_{1}\right\}
$$

Since $\psi$ is true on $\kappa$, there exist operations $\cdots G \cdots$ and relations $\cdots S \cdots$ on $\kappa$ so that $\varphi$ holds. Define operations $G_{S}$ and $G$ on $\kappa$ via

$$
\begin{aligned}
G_{S}(\vec{x}) & = \begin{cases}F_{0} & \text { if } \vec{x} \notin S, \\
F_{1} & \text { if } \vec{x} \in S,\end{cases} \\
G(x, y, z) & = \begin{cases}F_{0} & \text { if } x=y \neq z, \\
F_{1} & \text { if } x \neq y=z, \\
\text { anything in the remaining cases. }\end{cases}
\end{aligned}
$$

It should be clear that $\left(\kappa ; \cdots F \cdots, \cdots F_{R} \cdots, \cdots G \cdots, \cdots G_{S} \cdots\right.$, $G) \vDash \varphi^{*}$, and so we are done.

Conversely, suppose that $\kappa \vDash \psi^{\prime}$. Let us prove that $\kappa \vDash \psi$. Given operations $\cdots F \cdots$ and operations $\cdots R \cdots$, define operations $F_{0}, F_{1}$ and $\cdots F_{R} \cdots$ by first picking $F_{0} \neq F_{1}$ (and otherwise arbitrary) and then defining

$$
F_{R}(\vec{x})= \begin{cases}F_{0} & \text { if } \vec{x} \notin R, \\ F_{1} & \text { if } \vec{x} \in R .\end{cases}
$$

Since $\psi^{\prime}$ is true on $\kappa$, there exist operations $\cdots G, \cdots, \cdots G_{S} \cdots$ and $G$ on $\kappa$ so that $\varphi_{1} \rightarrow \varphi_{2}$ holds. Now clearly we already have $\varphi_{1}$ true, thus $\varphi_{2}$ holds. Since $F_{0} \neq F_{1}$ we know that $\varphi^{*}$ holds and that

$$
\forall x_{1} x_{2} \ldots\left[G_{S}\left(x_{1}, x_{2}, \ldots\right)=F_{0} \vee G_{S}\left(x_{1}, x_{2}, \ldots\right)=F_{1}\right]
$$

for each relation symbol $S$. If we define relations $\cdots S \cdots$ via $\vec{x} \in S \leftrightarrow G_{S}(\vec{x})$ $=F_{1}$, then it is easily checked that $\varphi$ is an easy consequence of $\varphi^{*}$ (using (*)).

Proof of The TheOrem Resumed. By Lemma 2 , our $\Pi_{2}^{1}$ class $X$ is defined by a second order sentence

$$
\psi=(\forall \cdots F \cdot \cdots)(\exists \cdots G \cdots)\left(\varphi_{1} \rightarrow \varphi_{2}\right),
$$

with $\varphi_{1}$ given by

$$
\forall a_{1} \cdots a_{M} \bigvee_{i=1}^{N} \bigwedge_{k=1}^{M} \alpha_{i k}=\beta_{i k}
$$

and $\varphi_{2}$ given by

$$
\forall x_{1} \cdots x_{N} \bigwedge_{j=1}^{N} \bigvee_{k=1}^{N} \gamma_{j k}=\delta_{j k} .
$$

Here we have deliberately chosen disjunctive normal form in one case, and conjunctive in the other, and we have taken $N$ as a single upper index large enough to cover all contingencies, and $M$ is even larger (and will be increased below). The 
$\gamma_{j k}$ and $\delta_{j k}$ are terms in $x_{1}, x_{2}, \ldots$, while the $\alpha_{i k}$ and $\beta_{i k}$ are terms in $a_{1}, a_{2}, \ldots$ This distinction will presently be important, when the $a_{i}$ will appear as (unknown) constant functions of $T$ and the $x_{i}$ will appear as variables of $T$. Moreover, we know that none of the operations $\cdots G \cdots$ appear in any of the equations $\alpha_{i k}=\beta_{i k}$.

We now claim that we may assume that among the $\cdots F \cdots$ we have $F_{0}, F_{1}$ (nullary), $F_{2}$ (quaternary) and $F_{3}(2 N$-ary) with these special properties:

(a) $F_{0}-F_{3}$ do not appear in $\varphi_{2}$.

(b) $\varphi_{1}$ entails these formulas:

$$
\begin{gathered}
F_{2}(x, x, y, z)=y, \quad F_{2}\left(F_{0}, F_{1}, y, z\right)=z, \\
x_{i}=x_{N+i} \rightarrow F_{3}\left(x_{1}, \ldots, x_{2 N}\right)=F_{0} \quad(1 \leqslant i \leqslant N) .
\end{gathered}
$$

(c) Any model of $\varphi_{1}$ remains a model of $\varphi_{1}$ upon altering $F_{0}-F_{3}$, so long as formulas (b) continue to hold.

Of course the claim is easily established by simply adding new operations and laws to $\varphi_{1}$ (thereby increasing $M$ ). To see that $\{\kappa: \psi \neq \kappa\}$ is unaltered, it is enough to observe that formulas (b) can be modeled on every set.

Abbreviation: $\alpha_{i}$ will stand for $\left(\alpha_{i 1}, \ldots, \alpha_{i M}\right)$; similarly $\beta_{i}, \gamma_{j}$ and $\delta_{j}$.

Definition. $T$ will consist of these terms:

$$
\begin{aligned}
\tau_{F} & =F\left(x_{1}, x_{2}, \ldots\right) \quad(\operatorname{each} F \text { of } \psi), \\
\tau_{i j} & =K_{i j}\left(x_{1}, \ldots, x_{N}, \alpha_{i}, F_{3}\left(\gamma_{j}, \delta_{j}\right)\right), \\
\tau_{i j}^{\prime} & =K_{i j}\left(x_{1}, \ldots, x_{N}, \beta_{i}, F_{1}\right),
\end{aligned}
$$

with $1 \leqslant i, j \leqslant N$. The variables of $T$ are expressly taken to be $x_{1}, \ldots, x_{N}$, and the "unknown" function symbols of $T$ are $\cdots F \cdots$ (explicitly appearing), $\cdots G \cdots$ (occurring in $\gamma_{j k}$ and $\left.\delta_{j k}\right),(N+M+1)$ )-ary $K_{i j}$ (new), and the nullary symbols $a_{1}, \ldots, a_{M}$ (occurring in $\alpha_{i k}$ and $\beta_{i k}$ ).

The recursiveness assertion of the Theorem is immediate, and so we now investigate the class of cardinals $\kappa \geqslant 2$ for which $T$ is universal in power $\kappa$. First let us assume that $\kappa \vDash \psi$ and prove that $T$ is universal in power $\kappa$.

Thus we are given operations $\cdots F \cdots, F_{i j}$ and $F_{i j}^{\prime}$ on $\kappa$ which we must simultaneously represent with the terms $\cdots \tau_{F} \cdots, \tau_{i j}$ and $\tau_{i j}^{\prime}$. This obviously amounts to saying that we are given operations $\cdots F \cdots$ and must find operations $\cdots G \cdots, a_{i}$, and $K_{i j}$ so as to represent $F_{i j}$ by $\tau_{i j}$ and $F_{i j}^{\prime}$ by $\tau_{i j}^{\prime}$. Looking at the form of $\tau_{i j}$ and $\tau_{i j}^{\prime}$, we see that each of the equations $\tau_{i j}=F_{i j}$ and $\tau_{i j}^{\prime}=F_{i j}^{\prime}$ is simply a definition of the operation $K_{i j}$ on a subset of its domain. Specifically, $K_{i j}$ is defined on these two subsets of its domain:

$$
A_{i j}=\left\{\left(x, \alpha_{i}, F_{3}\left(\gamma_{j}, \delta_{j}\right)\right): x \in \kappa^{N}\right\}, \quad B_{i j}=\left\{\left(x, \beta_{i}, F_{1}\right): x \in \kappa^{N}\right\} .
$$

To complete the proof of $\kappa$-universality, we need to see that $a_{1}, \ldots, a_{M}$ and operations $\cdots G \cdots$ can be chosen so that, for all $i$ and $j, A_{i j} \cap B_{i j}=\varnothing$.

Case 1. $(\kappa ; \cdots F \cdots) \vDash \varphi_{1}$. In particular, the formulas (b) hold. For $\kappa \geqslant 2$, the first two identities imply $F_{0} \neq F_{1}$. Moreover, the truth of $\psi$ tells us that we can find operations $\cdots G \cdots$ so that $(\kappa ; \cdots F \cdots, \cdots G \cdots) \vDash \varphi_{2}$. Thus for each 
$j$ and each $x \in \kappa^{N}$, we have $\gamma_{j 1}=\delta_{j 1}$ or $\gamma_{j 2}=\delta_{j 2}$ or $\cdots$ or $\gamma_{j N}=\delta_{j N}$. Hence by (b) we have $F_{3}\left(\gamma_{j}, \delta_{j}\right)=F_{0}$, and thus for each $i$ and $j$,

$$
A_{i j}=\left\{\left(x, \alpha_{i}, F_{0}\right): x \in \kappa^{N}\right\}
$$

which is disjoint from $B_{i j}$ (since $F_{0} \neq F_{1}$ ), regardless of our choice of $a_{1}, \ldots, a_{M}$.

Case 2. $(\kappa ; \cdots F \cdots) \vDash \neg \varphi_{1}$. Then we may choose $a_{1}, \ldots, a_{M}$ so that, for each $i, \alpha_{i} \neq \beta_{i}$. Clearly this makes $A_{i j}$ disjoint from $B_{i j}$ for all $i$ and $j$, regardless of our choice of the operations $\cdots G \cdots$. This completes our proof that $T$ is universal in power $\kappa$.

Conversely, let us suppose that $T$ is universal in power $\kappa \geqslant 2$ and prove that $\kappa \vDash \psi$. Thus we are given operations $\cdots F \cdots$ on $\kappa$ obeying $\varphi_{1}$, and we wish to find operations $\cdots G \cdots$ so that $\varphi_{2}$ holds. By (a) and (c), we may change $F_{0}-F_{3}$ to the following functions:

$F_{0}, F_{1}$ any two distinct constants;

$$
\begin{aligned}
& F_{2}(x, y, z, w)= \begin{cases}z & \text { if } x=y, \\
w & \text { if } x \neq y ;\end{cases} \\
& F_{3}\left(x_{1}, \ldots, x_{2 N}\right)= \begin{cases}F_{0} & \text { if } x_{1}=x_{N+1} \text { or } x_{2}=x_{N+2} \text { or } \cdots \text { or } x_{N}=x_{2 N}, \\
F_{1} & \text { otherwise. }\end{cases}
\end{aligned}
$$

Since $T$ is universal in power $\kappa$, there exist operations $\cdots F \cdots, \cdots G \cdots, a_{i}$ and $K_{i j}$ so that

$\tau_{F}=$ the given $\cdots F \cdots$,

$\tau_{i j}=F_{0} \tau_{i j}^{\prime}=F_{1}$.

Thus the "found" operations $\cdots F \cdots$ of our solution are none other than the given $\cdots F \cdots$. Moreover, referring to our previous analysis, the fact that $F_{0} \neq F_{1}$ tells us that we must have $A_{i j}$ disjoint from $B_{i j}$ for each $i$ and $j$. By $\varphi_{1}$, we have $\alpha_{i}=\beta_{i}$ for some $i$. Thus for this $i$ and each $j$, we can have $A_{i j}$ disjoint from $B_{i j}$ only if $F_{3}\left(\gamma_{j}, \delta_{j}\right) \neq F_{1}$ universally in $x_{1}, \ldots, x_{N}$. By our description of $F_{3}$ above, we see that this last inequality entails $\left(\gamma_{j 1}=\delta_{j 1}\right) \vee \cdots \vee\left(\gamma_{j N}=\delta_{j N}\right)$ for each $j$, universally in $x_{1}, \ldots, x_{N}$. Thus we have accomplished our goal of making $\varphi_{2}$ true in $(\kappa ; \cdots F \cdots, \cdots G \cdots)$ Q.E.D.

2. Remarks and examples. The first obvious remark allows us to pay no further attention to our requirement that $0,1 \in X$, since we may obviously find a first order sentence with models in just these two powers.

2.0. The class of all $\Pi_{2}^{1}$ classes is closed under finite unions and intersections, but not under complementation. For example $\left\{2^{\aleph_{0}}\right\}$ is not a $\Pi_{2}^{1}$ class, but its complement is. (See Garland [14] and Kunen [22]. Kunen's result is really a consistency result: it is consistent with ZFC that $\left\{2^{\kappa_{0}}\right\}$ is not $\Pi_{2}^{1}$, but it might be $\Pi_{2}^{1}$ anyway, for some accidental reason such as $\mathrm{CH}$, which forces $\left\{2^{\boldsymbol{\kappa}_{0}}\right\}=\left\{\boldsymbol{N}_{1}\right\}$, a $\Pi_{2}^{1}$ class.)

For our next two remarks we notice that the notion of $\Pi_{2}^{1}$ class can be enlarged to cover classes defined by infinitary sentences of pure second order logic of the form

$$
\psi=\forall \cdot R \cdot \cdots \exists \cdots S \cdots \bigwedge_{\alpha} \varphi_{\alpha}
$$

with possibly infinitely many $R$ 's or infinitely many $S$ 's or infinitely many $\alpha$ 's. 
2.1. An obvious extension of the proof in $\S 1$ shows that the classes (containing 0 , 1) definable in this way with finitely many $R$ 's are precisely the classes definable by universality of infinite sets $T$ of terms; in fact we will obtain $|T|<\aleph_{0}+|\psi|$.

2.2. If $\psi$ is recursively given, then the $\Pi_{2}^{1}$ class defined by $\psi$ is equal to the $\Pi_{2}^{1}$ class defined by some finite sentence, except possibly for finite cardinals. One needs to include a new predicate $N$, new operations $0, S,+$ on $N$, and add the axioms for " $Q$ " relativized to $N$. Here $Q$ is a finite fragment of Peano's axioms which is strong enough to provide a definition of every recursive function (see e.g. [46, p. 51]). One can then code $\psi$ as a finite sentence; for details of this procedure (and some other related procedures) see Kleene [19], and Craig and Vaught [5].

We can now examine some naturally occurring examples of $\Pi_{2}^{1}$ classes (sometimes in the form of 2.1 or 2.2 above).

2.3. The class of cardinals $\kappa$ not omitting a type $\Sigma$ (for some theory $\Gamma$ ). See Morley [33] or Proposition 7.2.4 on p. 434 of [4]. Morley's results yield the cardinal classes $\left(\beth_{\alpha}, \infty\right)$ for $\alpha$ any countable ordinal; for $\alpha$ recursive we get $\left(\beth_{\alpha}, \infty\right)$ given by a finite $\psi$, via 2.2 .

2.4. Two cardinal problems. Let $\Sigma$ be a first order theory in relations $\cdots T \cdot$. with a distinguished unary relation $U$. We say that $\Sigma$ admits $(\kappa, \lambda)$ if there exists a model $(A ; U, \ldots)$ of $\Sigma$ with $|A|=\kappa$ and $|U|=\lambda$. Suppose we are given a second order sentence

$$
\psi=\forall \cdots R \cdot \cdots \exists \cdots S \cdots \varphi
$$

which defines a class $X$ of cardinals. We are interested in the class $X^{\prime}$ defined by

$$
\psi^{\prime}=\forall U \cdots T \cdot R \cdots \exists \cdots S \cdots\left(\Sigma \rightarrow \varphi^{U}\right),
$$

with $\varphi^{U}$ denoting the relativization of $\varphi$ to $U$. It is routine to check that

$$
X^{\prime}=\{\kappa: \forall \lambda(\Sigma \text { admits }(\kappa, \lambda) \rightarrow \lambda \in X)\} .
$$

See Proposition 3.2.11(iv) on p. 133 of [4], and also Exercise 3.2.15, where one will find a finite $\Sigma$ which admits $(\kappa, \lambda)$ iff $\lambda \leqslant \kappa \leqslant \aleph_{n}(\lambda)$. One may easily check that in this case if $X=(\nu, \infty)$ then $X^{\prime}=\left(\aleph_{n}(\nu), \infty\right)$.

For another example take $\Sigma$ to say, of $(A ; U, T)$, that $T$ maps the $n$-element subsets of $A$ into $U$ in such a manner that there does not exist any $(n+1)$-element subset all of whose $n$-element subsets are mapped to the same place. The "arrow relation" theory of Erdõs and Hajnal (Theorem 39(i) of [12] and Lemma 5F of [11], or Theorem 7.2.1 of [4]) says that $\Sigma$ admits $(\kappa, \lambda)$ iff $\lambda<\kappa \leqslant z_{n}(\lambda)$. Taking $X$ as above, we obtain $X^{\prime}=\left(Z_{n}(\nu), \infty\right)$.

2.5 Rigid models of a theory. Let $\varphi_{1}$ be any first order sentence (or recursive theory) in relations $\cdots R \cdots$ and operations $\cdots F \cdots$, and consider the second order sentence

$$
\psi=\forall \cdots F \cdot R \cdot \exists \exists G_{1} G_{2}\left(\varphi_{1} \rightarrow \varphi_{2}\right),
$$

where $\varphi_{2}$ is the conjunction of the universal closures of these formulas:

$$
\begin{aligned}
G_{1} G_{2} x & =G_{2} G_{1} x=x, \\
G_{1} F\left(x_{1}, x_{2}, \ldots\right) & =F\left(G_{1} x_{1}, G_{1} x_{2}, \ldots\right) \quad(\text { each } F), \\
R\left(x_{1}, x_{2}, \ldots\right) & \leftrightarrow R\left(G_{1} x_{1}, G_{1} x_{2}, \ldots\right) \quad(\text { each } R), \\
& \exists x\left(G_{1} x \neq x\right) .
\end{aligned}
$$


Clearly $G_{1}$ represents a nonidentity automorphism of a model of $\varphi_{1}$. Thus $\kappa \vDash \psi$ iff $\varphi_{1}$ has no rigid models of power $\kappa$. (Obviously there is a corresponding $\psi$ for endomorphism-rigidity.)

Many examples of rigid spectra have been given by Ehrenfeucht [6] and then by Shelah [40]. Shelah obtains almost every $\Pi_{2}^{1}$ class as the complement of a rigid spectrum.

2.6. Simple algebras, etc. We may proceed as in 2.5 , but take $\varphi_{2}$ to say that certain relations define, e.g., a nontrivial homomorphic image, a nontrivial factorization, an onto endomorphism which is not one-one, and so on. In this way we obtain a $\Pi_{2}^{1}$ class $X$ whose complement is the class of powers of:

$$
\begin{aligned}
& \text { the simple algebras in } V \quad \text { (see, e.g., }[30],[23]) \text {, } \\
& \text { the Hopfian algebras in } V \text { (see }[24],[25]), \\
& \text { the directly indecomposable algebras in } V \text {, etc. }
\end{aligned}
$$

$\left(V=\operatorname{Mod} \varphi_{1}\right)$. Enlarging $\cdots F \cdots$ to include two new constants $F_{0}, F_{1}$, and taking $\varphi_{2}$ to say that $F_{0}=F_{1}$ or $S$ is a nontrivial congruence separating $F_{0}$ and $F_{1}$, we obtain the complement of the class of powers of subdirectly irreducible models of $\varphi_{1}$, a class which was studied in [47].

Pseudosimplicity [32] may also be handled in like manner.

2.7. Jónsson algebras. A Jónsson algebra is an infinite algebra with no proper subalgebras of the same power. See pp. 469-470 of Chang and Keisler [4], or pp. 120-135 of Jónsson [18] for a survey of this topic, which by and large remains mysterious and caught up with axiomatic questions in set theory. We can summarize, as follows, what is known about the existence of Jónsson algebras.

(1) One unary operation: they exist in power $\aleph_{0}$ only.

(2) $N$ unary operations $\left(2 \leqslant N \leqslant \aleph_{0}\right)$ : they exist in powers $\kappa_{0}$ and $\aleph_{1}$ only.

(3) $\lambda$ unary operations $\left(\lambda \geqslant \aleph_{1}\right)$ : they exist in powers $\aleph_{0}, \aleph_{1}, \ldots, \lambda^{+}$only.

(4) (Erdõs and Hajnal) If there exists a Jónsson algebra of any countable type in power $\kappa$, then there also exists a Jónsson algebra $(\kappa ; F)$ with $F$ binary.

(5) (Galvin, Rowbottom, Erdős, Hajnal, Chang) One binary operation: if there exists a Jónsson algebra of power $\kappa$, then there exists one of power $\kappa^{+}$; in particular they exist in power $\boldsymbol{\kappa}_{0}, \boldsymbol{\kappa}_{1}, \boldsymbol{\kappa}_{2}, \boldsymbol{\kappa}_{3}, \ldots$

(6) (Keisler, Rowbottom) One binary operation: if $V=L$, then they exist in all powers.

(7) (Erdõs, Hajnal) One binary operation: if $\mathrm{GCH}$, then for all $\kappa$ there is a Jónsson algebra of power $\kappa^{+}$. (Refined by Shelah in [39].)

(8) (Erdõs, Hajnal) One binary operation: if $(\kappa, F)$ is a Jónsson algebra, then $\kappa$ is not measurable.

Clearly the nonexistence of a Jónsson algebra of power $\kappa$ modeling $\varphi_{1}$ is described by a $\Pi_{2}^{1}$ class of cardinals, in a manner that should by now be familiar, thereby leading to a finite set $T$ of terms which is universal in power $\kappa>2$ iff there is no Jónsson algebra of power $\kappa$ modeling $\varphi_{1}$. For $\operatorname{Mod} \varphi_{1}=$ all algebras $(A ; F), F$ binary, this is one of the rare cases where we can write such a $T$ simply and directly. It consists of these eleven terms: 
$0, \quad 1, \quad F x y, \quad H x y, \quad J x y z w, \quad K_{1}(x, a, J a a c d, J 01 c d, H a a), \quad K_{1}(x, \varphi x, c, d, 0)$,

$$
\begin{gathered}
K_{2}(x, 1, \text { Jaacd, J01cd, Haa }), \quad K_{2}(x, H(\psi \varphi x, x), c, d, 0), \\
K_{3}(x, y, 1, \text { Jaacd, J01cd, Haa }), \quad K_{3}(x, y, H(\varphi G x y, F(\varphi x, \varphi y)), c, d, 0) .
\end{gathered}
$$

First, assume these terms are universal in $\kappa$; we will show that no $(\kappa ; F)$ can be a Jónsson algebra. To do this, we will apply the definition to this $F(x, y)$, to 0 and 1 any two distinct elements, to

$$
J x y z w=\left\{\begin{array}{ll}
z & \text { if } x=y, \\
w & \text { if } x \neq y,
\end{array} \quad H x y= \begin{cases}0 & \text { if } x=y, \\
1 & \text { if } x \neq y,\end{cases}\right.
$$

and with the pair $K_{1}(\cdots), K_{1}(\cdots)$ taken to be any two functions which never agree (e.g. the constant functions 0 and 1); likewise for the pairs $\left(K_{2}(\cdots)\right.$, $\left.K_{2}(\cdots)\right)$ and $\left(K_{3}(\cdots), K_{3}(\cdots)\right)$. Take $a, c, d, \varphi, \psi, K_{1}, K_{2}, K_{3}$ as supplied by universality. We obviously have

$$
\text { Jaacd }=c, \quad J 01 c d=d, \quad H a a=0,
$$

and so if the $K_{1}$-pair, the $K_{2}$-pair and the $K_{3}$-pair are each to disagree completely, we must have, for all $x$ and $y$ :

$$
\varphi x \neq a, \quad H(\psi \varphi x, x) \neq 1, \quad H(\varphi G x y, F(\varphi x, \varphi y)) \neq 1 .
$$

Referring to the way we chose $H$ above, we have, for all $x$ and $y$,

$$
\varphi x \neq a, \quad \psi \varphi x=x, \quad \varphi G x y=F(\varphi x, \varphi y) .
$$

These formulas tell us that $\varphi$ is a one-one mapping onto a proper subalgebra of $(\kappa ; F)$; thus $(\kappa ; F)$ is not a Jónsson algebra.

Conversely, let us assume there is no Jónsson algebra of power $\kappa$ and prove universality on $\kappa$. Given eleven operations corresponding to these terms, they first give us $F, H, J, 0$ and 1 . Now if (*) should fail for any $a, c, d$, then we would be done, since each $K_{i}$-pair would be defined on disjoint subsets. Thus we may assume that $(*)$ holds identically, and, in particular, that $0 \neq 1$. Since $(\kappa ; F)$ is not a Jónsson algebra, there exist $a, \varphi, \psi, G$ satisfying (**); from (*) we see

$$
\varphi x \neq a, \quad H(\psi \varphi x, x)=0, \quad H(\varphi G x y, F(\varphi x, \varphi y))=0 .
$$

Again we have each $K_{i}$-pair defined on disjoint subsets, and universality is assured.

Thus this set of eleven terms is universal in a somewhat mysterious set of cardinals, partly described by points $4-8$ above. Obviously similar sets can be obtained for the Jónsson algebras in any finitely based variety. Shelah recently found an uncountable Jónsson group (see [41]), but almost nothing is known in general (but cf. [29], [20] and [21]).

2.8. Examples from set theory, higher cardinals, etc. The reader is invited to try his hand at representing these as $\Pi_{2}^{1}$ classes:

strong limit cardinals,

strongly inaccessible cardinals,

Ramsey cardinals,

inaccessible weakly compact cardinals. 
The class of measurable cardinals is not so representable, as W. N. Reinhardt has informed us, for it is impossible to define measurability of $\kappa$ without reference to the power set of $\kappa$, or something else at least as large.

All of these may of course be converted to a finite set of terms in the manner of the Main Theorem. But in one case, regularity, it is possible to give a nice simple $T$ directly. Namely, $\kappa$ is regular iff this set of terms is universal in power $\kappa$ :

$$
\begin{gathered}
0, \quad 1, \quad E x y, \quad S x, W x, H x y z w, J x y z w, \\
K(x, 1, J a a c d, J 01 c d, H a a c d, H a b c c, a), \\
K(x, H(x, R S T x, x, \operatorname{Re}(S a, T x)), c, d, 0,0, E(S a, W a)) .
\end{gathered}
$$

The proof is similar to that in 2.7 .

2.9. The finite range. A good many examples are known concerning universality of $T$ in power $\kappa<\omega$, even for $T$ a single term in unary operations; consult the papers of Isbell, Ehrenfeucht and Silberger. Some other interesting examples can be found earlier in this section, for instance, the set of all integers $k$ which are not the order of any finite simple group (2.6).

First order spectra play an interesting role in the finite range (although, by the Skolem-Löwenheim theorem, they do not discriminate between any infinite cardinals). If $\varphi_{1}$ is a first order sentence in $\cdots F \cdots$ and $\cdots R \cdots$, and $\varphi_{2}$ is a first order sentence in $\cdots G \cdots$ and $\cdots S \cdots$, then $(\forall \cdots F \cdots R \cdots)(\exists \cdots G \cdots S \cdots)\left(\varphi_{1} \rightarrow \varphi_{2}\right)$ holds precisely on Spec $\varphi_{2}$ $\cup\left(\right.$ Complement $\left.\operatorname{Spec} \varphi_{1}\right)$. For $\varphi_{1}=\top$, we get $\operatorname{Spec} \varphi_{2}$, and for $\varphi_{2}=\perp$, we get $\left(\right.$ Complement Spec $\left.\varphi_{1}\right) \cup\{0,1\}$.

Again we may bypass the Main Theorem in one special case, this time representing $\{0,1\} \cup\left(\right.$ Complement $\left.\operatorname{Spec} \varphi_{1}\right)$ as $\{\kappa: T$ is universal in power $\kappa\}$ for a very easily described $T$. We will assume that $\varphi_{1}$ is the conjunction of identities $\sigma_{i}=\tau_{i}$ $(1 \leqslant i \leqslant N)$. The following terms are obviously universal in power $\kappa$ iff $\kappa=0$ or 1 or $\kappa \notin \operatorname{Spec} \varphi_{1}$ :

$$
T\left\{\begin{array}{l}
F\left(x_{1}, x_{2}, \ldots\right) \quad\left(\text { any } F \text { of } \varphi_{1}\right), \\
K\left(\sigma_{1}\left(a_{1}, a_{2}, \ldots\right), \ldots, \sigma_{N}\left(a_{1}, a_{2}, \ldots\right)\right), \\
K\left(\tau_{1}\left(a_{1}, a_{2}, \ldots\right), \ldots, \tau_{N}\left(a_{1}, a_{2}, \ldots\right)\right)
\end{array}\right.
$$

By the way, this construction of $T$ makes the Corollary obvious. For $T$ is obviously recursive in $\varphi_{1}$, and it is undecidable whether $\omega \in \operatorname{Spec} \varphi_{1}$ (by Perkins [35]).

2.10. Unions, revisited. If $T$ and $T^{\prime}$ are finite sets of terms, then the Main Theorem (together with 2.0) tells us that $\{\kappa: T$ universal in $\kappa\} \cup\left\{\kappa: T^{\prime}\right.$ universal in $\kappa\}$ must be of the form $\left\{\kappa: T^{\prime \prime}\right.$ universal in power $\left.\kappa\right\}$ for some finite set $T^{\prime \prime}$, but it is not immediately apparent how to get a simple description of $T^{\prime \prime}$. In the special case $T=\{\tau(x, y)\}$ and $T^{\prime}=\{\sigma(u, v)\}$ (with no function symbols appearing in both), the reader may enjoy checking that the following will do for $T^{\prime \prime}$ :

$$
\begin{gathered}
0, \quad 1, \quad J x y u v, \quad H x y u v, \quad F x y, \quad G u v, \\
K(x, y, u, v, 1, H a a c d, H a b c c, \text { Jaacd, J01 } c d), \\
K(x, y, u, v, H(F x y, \tau(x, y), G u v, \sigma(u, v)), 0,0, c, d) .
\end{gathered}
$$


Problem. Is there a map $\left(T, T^{\prime}\right) \mapsto T^{\prime \prime}$ such that, for all clones $C, T^{\prime \prime}$ is universal on $C$ iff $T$ or $T^{\prime}$ is universal on $C$ ?

Our $T^{\prime \prime}$ works for the clones $C(\kappa)$, but seems to use special properties of those clones. Of course, if "or" is replaced by "and", the answer is obviously yes. ( $T$ " is a "disjoint union" of $T$ and $T^{\prime}$.)

2.11. It is fairly easy to get $\left[0, \aleph_{0}\right] \cup\left[2^{\aleph_{0}}, \infty\right)$ as a $\Pi_{2}^{1}$ class. Thus our Theorem gives us a finite set of terms which is universal in all powers iff $\mathrm{CH}$ is true.

3. A special example, not in the style of $\$ 1$. First, we should point out that Isbell discovered [15] that the single term $f^{2} g^{2} f x$ is universal in all finite powers but not in any infinite power.

Now we give an example which yields $\{0,1\} \cup\left[\boldsymbol{\aleph}_{0}, \infty\right)$ in a way that seems different from the examples that have come before. We claim that the set $f_{1} F x y$, $f_{2} F x y$ is universal in power $\kappa$ iff $\kappa=0,1$ or $\kappa \geqslant \kappa_{0}$. Certainly, if this set is universal, then there exist $F, f_{1}, f_{2}$ obeying $f_{1} F x y=x, f_{2} F x y=y$, which implies that $F: A^{2} \rightarrow A$ is one-one, and thus $|A| \leqslant 1$ or $|A| \geqslant \aleph_{0}$. On the other hand, assume $A$ is infinite and let $F: A^{2} \rightarrow A$ be any one-one map. It is then easy to see how to solve $f_{1} F x y=F_{1} x y, f_{2} F x y=F_{2} x y$ for $f_{1}$ and $f_{2}$.

\section{REFERENCES}

1. J. W. Addison, L. Henkin and A. Tarski (Editors), The theory of models, Proc. 1963 Sympos. (Berkeley, Calif.), North-Holland, Amsterdam, 1965.

2. J. T. Baldwin and Joel Berman, Elementary classes of varieties (to appear).

3. S. Banach, Sur un théorème de M. Sierpinski, Fund. Math. 25 (1935), 5-6.

4. C. C. Chang and H. J. Keisler, Model theory, North-Holland, Amsterdam, 1973.

5. W. Craig and R. L. Vaught, Finite axiomatizability using additional predicates, J. Symbolic Logic 23 (1958), 289-308.

6. A. Ehrenfeucht, Elementary theories with models without automorphisms, The Theory of Models, North-Holland, Amsterdam, 1965, pp. 70-76.

7. A. Ehrenfeucht, S. Fajtlowicz, J. Malitz and J. Mycielski, Some problems on the universality of words in groups, Algebra Universalis 11 (1980), 261-263.

8. A. Ehrenfeucht and D. M. Silberger, Decomposing a transformation with an involution, Algebra Universalis 7 (1977), 179-190.

9. $399-402$.

10. __ Universal terms of the form $B^{n} A^{m}$, Algebra Universalis 10 (1980), 96-116.

11. P. Erdös, A. Hajnal and R. Rado, Partition relations for cardinal numbers, Acta Math. Acad. Sci. Hungar. 16 (1965), 93-196.

12. P. Erdös and R. Rado, A partition calculus in set theory, Bull. Amer. Math. Soc. 62 (1956), 427-489.

13. R. Freese and R. McKenzie, Residually small varieties with modular congruence lattices, Trans. Amer. Math. Soc. 264 (1981), 419-430.

14. S. J. Garland, Second-order cardinal characterizability, Proc. Sympos. Pure Math., vol. 13, part 2 , Amer. Math. Soc., Providence, R. I., 1974, pp. 127-146.

15. J. Isbell, On the problem of universal terms, Bull. Acad. Polon. Sci. Sér. Sci. Math. 14 (1966), 593-595.

16. J. R. Isbell, M. I. Klun and S. H. Schanuel, Affine parts of algebraic theories. I, J. Algebra 44 (1977), 1-8.

17. B. Jonsson, The unique factorization problem for finite relational structures, Colloq. Math. 14 (1966), 1-32.

18. Topics in universal algebra, Lecture Notes in Math., vol. 250, Springer-Verlag, Berlin, 1972.

19. S. C. Kleene, Finite axiomatizability of theories in the predicate calculus using additional predicate symbols, Mem. Amer. Math. Soc. No. 10 (1952), 27-68. 
20. A. Kostinsky and R. McKenzie, Notices Amer. Math. Soc. 15 (1968), 389; Abstract \#68T-302.

21. A. Kostinsky, Some problems for rings and lattices within the domain of general algebra, Ph.D. Thesis, Berkeley, Calif., 1969.

22. K. Kunen, Indescribability and the continuum, Proc. Sympos. Pure Math., vol. 13, part 1, Amer. Math. Soc., Providence, R. I., 1971, pp. 199-203.

23. W. A. Lampe and W. Taylor, Simple algebras in varieties, Algebra Universalis (to appear).

24. J. T. Loats, Hopfian Boolean algebras of power < continuum, Proc. Amer. Math. Soc. 77 (1979), $186-190$.

25. J. T. Loats and M. Rubin, Boolean algebras without nontrivial endomorphisms exist in every uncountable cardinality, Proc. Amer. Math. Soc. 72 (1978), 346-351.

26. G. G. Lorentz, The thirteenth problem of Hilbert, Proc. Sympos. Pure Math., vol. 28, Amer. Math. Soc., Providence, R. I., 1976, pp. 419-430.

27. J. Loś, Un theorème sur les superpositions des fonctions définies dans les ensembles arbitraires, Fund. Math. 37 (1950), 84-86.

28. R. McKenzie, On elementary types of symmetric groups, Algebra Universalis 1 (1971), 13-20.

29. __ On semigroups whose proper subsemigroups have lesser power, Algebra Universalis 1 (1971),

21-25.

30. R. McKenzie and S. Shelah, The cardinals of simple models for universal theories, Proc. Sympos. Pure Math., vol. 25, Amer. Math. Soc., Providence, R. I., 1971, pp. 53-74.

31. G. McNulty, The decision problem for equational bases of algebras, Ann. Math. Logic 19 (1976), 193-259.

32. D. Monk, On pseudo-simple universal algebras, Proc. Amer. Math. Soc. 13 (1962), 543-546.

33. M. Morley, Omitting classes of elements, The Theory of Models, North-Holland, Amsterdam, 1965, pp. 265-273.

34. J. Myhill et al., Advanced Problems 6244, and solution, Amer. Math. Monthly 85 (1978), 828, and solution, 87 (1980), 676-678.

35. P. Perkins, Unsolvable problems for equational theories, Notre Dame J. Formal Logic 8 (1967), 175-185.

36. A. G. Pinus, Elementary definability of symmetry groups, Algebra Universalis 3 (1973), 59-66.

37. E. Redi, Representation of Menger systems by multiplace endomorphisms, Učen. Zap. Tartu Gos. Univ. 277 (1971), 47-51.

38. S. Shelah, The first order theory of permutation groups, Israel J. Math. 14 (1973), 149-162; Erratum, ibid. 15 (1973), 437-441.

39. __ Notes on combinatorial set theory, Israel J. Math. 14 (1973), 262-277.

40. ___ Refuting Ehrenfeucht conjecture on rigid models, Israel J. Math. 25 (1976), 273-286.

41. _ On a problem of Kurosh, Jónsson groups, and applications, Word Problems, Decision Problems and the Burnside Problem in Group Theory. II (Adjan, Boone and Higman, Editors) (to appear).

42. W. Sierpiński, Sur les suites infinies de fonctions définies dans les ensembles quelconques, Fund. Math. 24 (1934), 209-212.

43. D. M. Silberger, When is a term point-universal?, Algebra Universalis 10 (1980), 135-154.

44. __ Universal terms of complexity three, Algebra Universalis (to appear).

45. __ $B^{n} A^{m}$ is universal iff point-universal, Algebra Universalis (to appear).

46. A. Tarski, A. Mostowski and R. Robinson, Undecidable theories, North-Holland, Amsterdam, 1953, 1968, 1971.

47. W. Taylor, Residually small varieties, Algebra Universalis 2 (1972), 33-53.

48. ___ Equational logic, Houston J. Math. Survey Issue, 1979, iii +83 pp.

49. __ Laws obeyed by topological algebras-extending results of Hopf and Adams, J. Pure Appl. Algebra (to appear).

50. __ Abstracts Amer. Math. Soc. 1 (1980), 218, 390; Abstracts \# 80T-A61, \#80T-E57.

51. M. L. Valente, Sobre a univeralidade de palavras para grupos simétricos, MS Thesis, Florianópolis, 1979.

52. A. G. Vitushkin, On representation of functions by means of superpositions, and related topics, Enseign. Math. 23 (1977), 255-320.

53. P. Winkler, Polynomial hyperforms (to appear).

Department of Mathematics, University of Colorado, Boulder, Colorado 80309 